\title{
The Potential role of ACEi and ARBs in COVID-19; A Perspective
}

\author{
*Ahmad Hassan
}

\section{Summary:}

COVID-19 pandemic has caused significant morbidity and mortality around the world. The disease severity ranges from mild upper respiratory infection to severe lower respiratory and cardiac illness. Acute respiratory distress syndrome (ARDS) is the most serious complication and results in diffuse inflammatory alveolar damage, respiratory failure, and death. Components of the ReninAngiotensin-Aldosterone-System (RAAS) are involved in an inflammatory reaction in the lungs. Various studies have shown that blocking RAAS peptides in the lungs especially angiotensinconverting enzyme (ACE) and type-1 angiotensin receptor (ATR1) reduces lung injury, improves respiratory function and is associated with better clinical outcomes in the COVID-19 patients. We suggest that angiotensin-converting enzyme inhibitors (ACEi) and angiotensin receptor blockers (ARBs) - drugs that block RAAS peptides - be considered for a repurposed use in COVID-19 induced lung injury.

\section{Introduction:}

Coronavirus disease 2019 (COVID-19) emerged as a respiratory illness of unknown infectious origin in China. It then spread all around the world and has caused substantial mortality. The disease-causing virus, SARS-CoV2 (later identified), is closely related to other beta coronaviruses, SARS-CoV (2003) and MERS-CoV (2012), that have caused severe respiratory disease pandemics in the past two decades. The disease follows a variable pattern, ranging from asymptomatic or mild upper respiratory or gastrointestinal infection (1) to a severe lower respiratory tract (pneumonia) or cardiac (myocarditis) illness, especially in patients with other co-morbidities (2). In very severe cases, a hyperinflammatory response causes cytokine-storm leading to Acute Respiratory Distress Syndrome (ARDS), respiratory failure, sepsis, and death (3). The exact mechanism of this variable disease expression is not yet understood.

The pathogenicity and tissue tropism of a pathogen is determined by the type of interaction with the host receptors and the tissue distribution of interacting receptors in the host (4). SARS-CoV2 is genetically $72 \%$ similar to SARS-CoV and likewise interacts with Angiotensin-Converting Enzyme 2 (ACE2) as receptor through its spike glycoprotein (S) to bind, fuse and enter into the host cells (5) (6) (7). ACE2 is a component of the Renin-Angiotensin-Aldosterone-System (RAAS) and is expressed abundantly in airway epithelia, lung parenchyma, heart, intestines, esophagus, bladder, and kidney (5).

The RAAS regulates fluid balance and blood pressure through several peptides, including renin, angiotensin 2 (AT2) and aldosterone. The precursor angiotensinogen is released from the liver and is cleaved by renin into angiotensin 1 which is, in turn, converted into angiotensin 2 by the Angiotensin-Converting Enzyme 1 (ACE) in the lungs. Angiotensin 2 (AT2) maintains vascular tone, blood pressure and fluid volume through its vasoconstrictor effect, the release of aldosterone and vasopressin, and direct catecholaminergic effects on cardiac myocytes. AT2 is then converted into angiotensin (1-7) by Angiotensin-Converting Enzyme 2 (ACE2). Angiotensin (1-7) mitigates the effects of AT2 and thus a balance exists between ACE and ACE2 for appropriate homeostasis (8). 
Angiotensin-Converting Enzyme Inhibitors (ACEi) and Angiotensin Receptor Blockers (ARBs) are drugs that act on the RAAS and are used in hypertension, cardiomyopathies and heart failure. Much controversy exists on the effects of these drugs on the respiratory and cardiac pathology of COVID19 (9). Long term use of ACEi can upregulate ACE2 (5) due to the absence of its natural ligand AT2. The long-term use of ARBs has been also been associated with the upregulation of ACE2 (10). This may cause an increased number of ACE2 receptors made available to the virus for infecting host cells and consequently enhanced pathogenicity. Indeed, hypertension and cardiovascular diseases are the top two co-morbidities associated with the worst outcome in COVID-19 (2) and that may be attributed to the long term use of ACEis or ARBs.

\section{Role of RAAS Components in COVID-19 Lung Injury:}

In addition to their respective roles in RAAS, ACE, ACE2, and AT2 are also involved in the pathogenesis of acute lung injury. Interestingly, an imbalance between ACE and ACE2 activity is found in various disease models of ARDS (the most common complication of COVID-19). Increased ACE activity and decreased ACE2 activity in the lungs is associated with diffuse alveolar edema and lung injury (11). AT2 has been found to have proinflammatory and profibrotic effects (in addition to RAAS effects) (12) and lung levels of AT2 were found to be elevated in mice model of SARS-CoV (13) induced ARDS and in COVID-19 patients (14). ACE2 has been shown to protect mice from severe lung damage induced by acid aspiration (13). ACE2 also hydrolyzes and inactivates desbradykinin, a proinflammatory peptide (15), that can potentially worsen inflammation-induced lung damage. Blockade of Angiotensin Receptor 1 (ATR1) with Losartan attenuates acute lung injury in mouse models of ARDS (13). The lung-protective effects of ACEi and ARBs against lung injury in ARDS have been described in various clinical studies as well (16) (17). Accordingly, increased ACE2 activity and ATR1 antagonism have a protective role in acute lung injury and ARDS (18) (13).

A hypothesized mechanism of COVID-19 lung injury (based on studies on previous SARS-CoV) is that the SARS-CoV2 binds ACE2 in the lungs through the spike glycoprotein (S). S is cleaved at a polybasic site by transmembrane protease (TMPRSS2) into S1 and S2 (6), the virion fuses with the epithelial cell membrane and is internalized along with ACE2 by pH-dependent endocytosis (19). Internalization results in the downregulation of ACE2 (20), thus tipping the balance towards relative excess of ACE. This results in more angiotensin 2 and that may worsen lung injury and ARDS (Fig A).

Increased number of ACE2 receptors can cause increased viral load that causes more lung damage. This presents a conflicting paradox to the lung-protective effects of ACE2 and ATR1 blockade. It can be partly explained by the timing of expression (5) (21) of ACE2 receptors on airway epithelia and lung parenchyma. The preexisting increased numbers of ACE2 (due to long-term ACEi or ARB use) can provide a good homing ground to the SARS-CoV2 and may cause increased pathogenicity. Also, after interaction with the virus, the ACE2 would internalize, thus decreasing the number of available ACE2 at the time of lung injury that comes late after virus internalization. Contrarily, inducing ACE2 expression, or blocking ATR1 at the time of lung-injury may enhance the protective effects of these mechanisms. These mechanisms are not fully characterized and we suggest further experimental studies to determine the exact underlying mechanisms. 

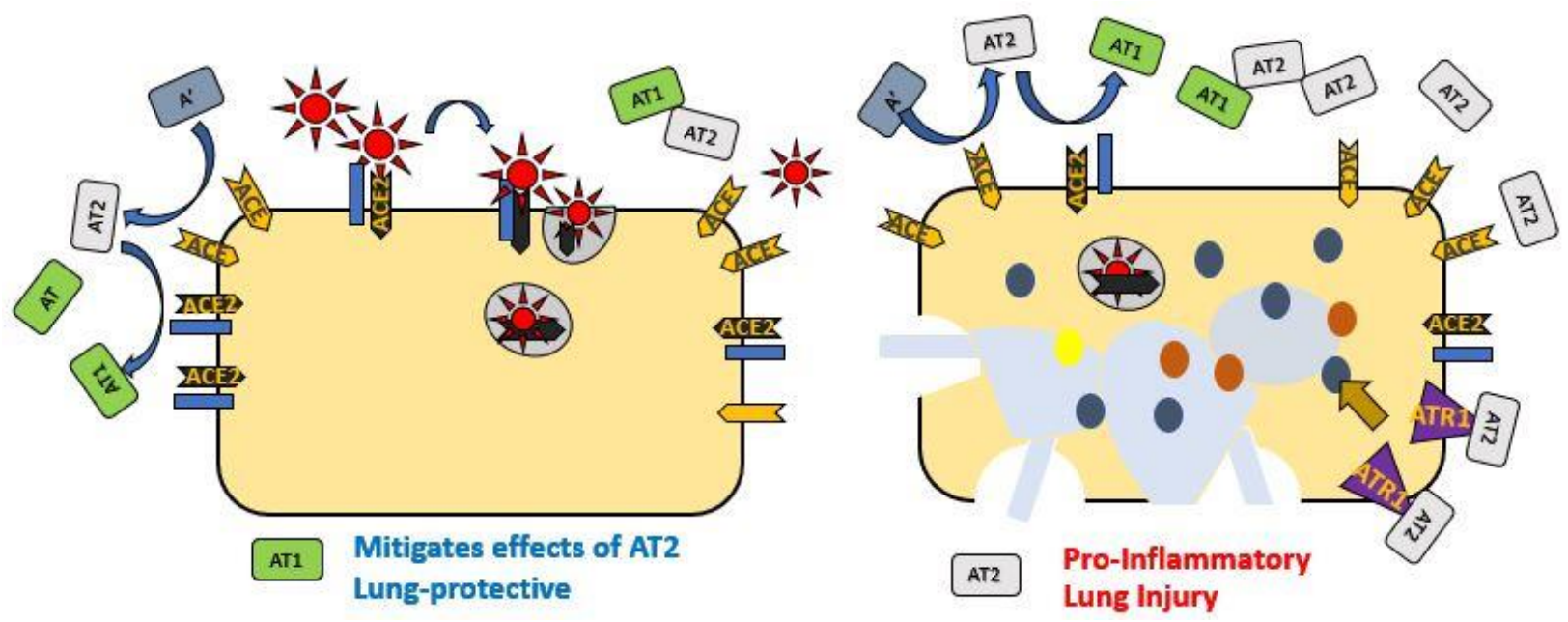

Fig A. Hypothesized mechanism of SARS-CoV2 cell-infectivity and subsequent lung-damage with focus on the balance between ACE (yellow) and ACE2 (black). SARS-CoV2 (red spiked spheres) via its spike glycoprotein $S$ bind with ACE2 on lung epithelial cells. $S$ is cleaved to $s 1$ and $s 2$ (not shown) by TMPRSS (blue stripe) and internalized. This also leads to the internalization of ACE2 and thus decreased numbers of ACE2 on the epithelium. Decreased ACE2 and relative increased ACE disturbs the balance. This subsequently results in more AT2 compared to AT1 (as shown in the second part of the figure). AT2 binds its receptor ATR1 and can potentially contribute towards diffuse alveolar edema and inflammatory acute lung injury as shown by edema and infiltrates in the second part of the figure. Red spiked spheres $=$ SARS-CoV2. Yellow chevron arrows $=$ ACE. Black chevron arrows $=$ ACE2 . Blue strips $=$ TMPRSS. Light blue box $=$ Angiotensin 2 (AT2). Green box = Angiotensin 1-7 (AT1). Purple triangle = Angiotensin 2 Receptor 1 (ATR1).

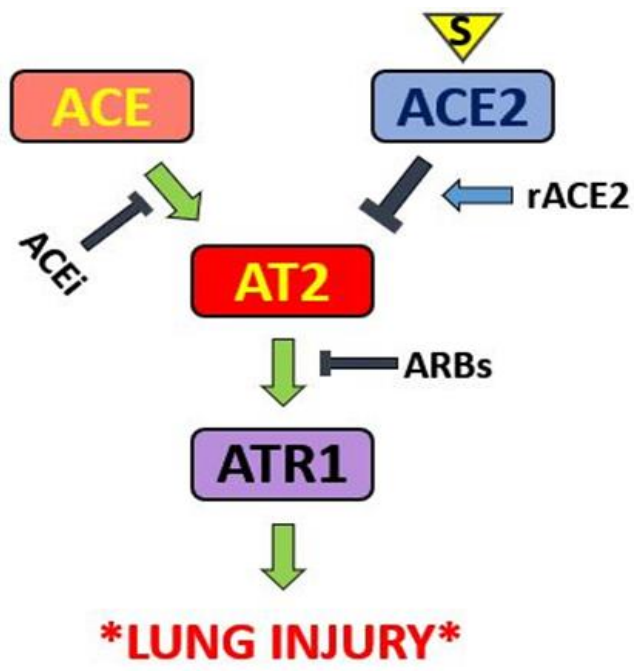

Fig B. Schematic role of RAAS components (ACE, ACE2, AT2, and ATR1) in acute lung injury and sites where RAAS antagonists (rACE2, ACEi, and ARBs) block the inflammatory pathway and attenuate inflammatory lung injury. $S$ (yellow triangle) = Spike Glycoprotein of SARS-CoV2. ACE2 counter balance ACE and subsequent pathway by deactivating AT2. rACE2 = recombinant ACE2, can potentiate activity of ACE2. 


\section{The Potential Lung-Protective Role of RAAS Antagonists in COVID-19:}

Based on the fact that angiotensin 2 (AT2) has proinflammatory activity in the lungs and the inflammatory basis of acute lung injury and ARDS in COVID-19 patients, the following strategies may protect against the fatal lung damage in COVID-19. (a) Therapeutically inducing ACE2 activity. (b) Blocking Lung Angiotensin Receptor 1 (ATR1). (c) Decreasing lung Angiotensin 2 (AT2) levels. (Fig. B). Recombinant ACE2 receptor can both block the virus from binding natural ACE2 and also counterbalance the proinflammatory effects of ACE. It has shown promising results in experimental studies (22) but clinical trials are yet to be completed. ACEi and ARBs are available drugs and their pharmacokinetics, pharmacodynamics, and safety profile are well established. These drugs can work by decreasing the AT2 levels and blocking ATR1 in the lungs, both effects rescue acute lung injury. A recent clinical study $(n=417)$ has demonstrated the beneficial effects of local ReninAngiotensin antagonism against inflammatory lung injury in COVID-19 patients. ARB and ACEi use was shown to be associated with less inflammatory lung injury, enhanced acquired immunity, decreased viral load, and better clinical outcomes in COVID-19 patients (23). We, therefore suggest that these drugs can be repurposed to be used in COVID-19 lower respiratory tract injury (8) after appropriate testing, dose adjustments and optimization.

It is worth mentioning here, that, although there has been an association between the use of ACEi/ARBs and the worst cardiorespiratory outcome in COVID-19, there is no adequate experimental or clinical evidence in humans yet that long-term use of ACEi or ARBs worsen the cardiorespiratory illness associated with SARS viruses (4) (24). Some further clinical trials are going on to ascertain and confirm the exact effects of Renin-Angiotensin antagonism on COVID-19 associated lung-injury and results are awaited (25) (NCT04330300). Current recommendations, however, are to continue the use of ACEi and ARBs for their renal and cardiac protective benefits (24).

\section{Conclusion:}

The proinflammatory role of RAAS components, ACE and AT2 in acute lung injury coupled with the inflammatory nature of lung damage in COVID-19, justifies that RAAS antagonists (ARBs and ACEi) can be considered as a therapeutic modality to protect against acute lung injury and respiratory failure in COVID-19. As these drugs are already in use, and their kinetics and dynamics are well known, they can be readily readopted for their lung-protective benefits. The dose and timing of administration of these drugs will need to be optimized.

\section{REFERENCES:}

1. Gu J, Han B, Wang J. COVID-19: Gastrointestinal Manifestations and Potential Fecal-Oral Transmission. Gastroenterology [Internet]. 2020;(April):118-9. Available from: https://doi.org/10.1053/j.gastro.2020.02.054

2. Zhou F, Yu T, Du R, Fan G, Liu Y, Liu Z, et al. Clinical course and risk factors for mortality of adult inpatients with COVID-19 in Wuhan, China : a retrospective cohort study. Lancet [Internet]. 2020;395(10229):1054-62. Available from: http://dx.doi.org/10.1016/S01406736(20)30566-3

3. Mehta P, Mcauley DF, Brown M, Sanchez E, Tattersall RS, Manson JJ, et al. COVID-19: consider 
cytokine storm syndromes and immunosuppression. Lancet [Internet].

2020;395(10229):1033-4. Available from: http://dx.doi.org/10.1016/S0140-

6736(20)30628-0

4. Maginnis MS. Virus-Receptor Interactions: The Key to Cellular Invasion. J Mol Biol [Internet]. 2018/06/18. 2018 Aug 17;430(17):2590-611. Available from:

https://pubmed.ncbi.nlm.nih.gov/29924965

5. Burchfield, J. Renin-Angiotensin-Aldosterone System: Double-Edged Sword in COVID-19 Infection. Preprints 2020, 2020030365.

6. Glycoprotein C-S, Walls AC, Park Y, Tortorici MA, Wall A, Mcguire AT, et al. Structure, Function, and Antigenicity of the SARS- Structure, Function, and Antigenicity of the SARSCoV-2 Spike Glycoprotein. Cell [Internet]. 2020;1-12. Available from: https://doi.org/10.1016/j.cell.2020.02.058

7. Structural basis for the recognition of SARS-CoV-2 by full-length human ACE2. 2020;2(March):1444-8.

8. South AM, Diz DI, Chappell MC. COVID-19, ACE2, and Cardiovascular Consequences. 2020;123.

9. Tignanelli CJ, Ingraham NE, Sparks MA, Reilkoff R, Bezdicek T, Benson B, et al. Correspondence Antihypertensive drugs. Lancet Respir [Internet]. 2020;2600(20):2019-20. Available from: http://dx.doi.org/10.1016/S2213-2600(20)30153-3

10. Igase M, Kohara K, Nagai T, Miki T, Ferrario CM. Increased Expression of AngiotensinConverting Enzyme 2 in Conjunction with Reduction of Neointima by Angiotensin II Type 1 Receptor Blockade. 2008;31(3):553-9.

11. Nicholls J, Peiris M. Good ACE, bad ACE do battle in lung injury, SARS. 2005;11(8):821-2.

12. Benigni A, Cassis P, Remuzzi G. Angiotensin II revisited: new roles in inflammation, immunology, and aging. 2010;247-57.

13. Kuba K, Imai Y, Rao S, Gao H, Guo F, Guan B, et al. A crucial role of angiotensin-converting enzyme 2 ( ACE2 ) in SARS coronavirus - induced lung injury. 2005;11(8):875-9.

14. Liu Y, Yang Y, Zhang C, Huang F, Wang F, Yuan J, et al. Clinical and biochemical indexes from 2019-nCoV infected patients linked to viral loads and lung injury. 2020;63(3):364-74.

15. Sodhi CP, Wohlford-Lenane C, Yamaguchi Y, Prindle T, Fulton WB, Wang S, et al. Attenuation of pulmonary ACE2 activity impairs inactivation of des-Arg(9) bradykinin/BKB1R axis and facilitates LPS-induced neutrophil infiltration. Am J Physiol Lung Cell Mol Physiol [Internet]. 2017/09/21. 2018 Jan 1;314(1): L17-31. Available from: https://pubmed.ncbi.nlm.nih.gov/28935640

16. Article O, Kim J, Choi SM, Lee J, Park YS, Lee CH, et al. Effect of Renin-Angiotensin System Blockade in Patients with Acute Respiratory Distress Syndrome : A Retrospective CaseControl Study. 2017;29(04):154-63.

17. Ruthman CA, Festic E. Emerging therapies for the prevention of acute respiratory distress syndrome. Ther Adv Respir Dis [Internet]. 2015/05/22. 2015 Aug;9(4):173-87. Available from: https://pubmed.ncbi.nlm.nih.gov/26002528 
18. Imai Y, Kuba K, Rao S, Huan Y, Guo F, Guan B, et al. Angiotensin-converting enzyme 2 protects from severe acute lung failure. 2005; 436(July).

19. Yang N, Shen H. Targeting the Endocytic Pathway and Autophagy Process as a Novel Therapeutic Strategy in COVID-19. 2020;16.

20. Nl C, Glowacka I, Bertram S, Herzog P, Pfefferle S, Steffen I, et al. Differential Downregulation of ACE2 by the Spike Proteins of Severe Acute Respiratory Syndrome Coronavirus and Human. 2010;84(2):1198-205.

21. Rockx B, Baas T, Zornetzer GA, Haagmans B, Sheahan T, Frieman M, et al. Early Upregulation of Acute Respiratory Distress Syndrome-Associated Cytokines Promotes Lethal Disease in an Aged-Mouse Model of Severe Acute Respiratory Syndrome Coronavirus Infection 0. 2009;83(14):7062-74.

22. Monteil V, Kwon H, Prado P, Hagelkrüys A, Wimmer RA. Inhibition of SARS-CoV-2 infections in engineered human tissues using clinical-grade soluble human ACE2. 2020;

23. Meng J, Xiao G, Zhang J, He X, Ou M, Bi J, et al. Renin-angiotensin system inhibitors improve the clinical outcomes of COVID-19 patients with hypertension. 2020;1751.

24. Angiotensin- PI. Cardiovascular Disease : A Viewpoint on the Enzyme Inhibitors/ Angiotensin Receptor. 2020;2019:1-5.

25. ClinicalTrials.gov [Internet]. John William McEvoy (MBBCh, MHS): National Library of Medicine (US). 2020 Apr 01 -. Identifier NCT04330300, Coronavirus ACEi/ARB Investigation (CORONACION); 2020 Apr 01. Available from:

https://clinicaltrials.gov/ct2/show/NCT04330300

Author: Ahmad Hammad Hassan (MBBS, M.Phil).

Medical Officer at Primary and Secondary Healthcare Department, Punjab, Pakistan.

Email: ahmadhammadhassan@gmail.com 